\title{
Potential Inhibitory Effect of LASSBio-596, a New Thalidomide Hybrid, on Inflammatory Corneal Angiogenesis in Rabbits
}

\author{
Joao Crispim M.L. Ribeiro ${ }^{a} \quad$ Francisco Vagnaldo Fechine $^{a}$ \\ Mariana Zaira M.L. Ribeiro ${ }^{a}$ Eliezer J. Barreiro ${ }^{b} \quad$ Lidia Moreira Lima $^{b}$ \\ Nagila M.P.S. Ricardo ${ }^{a}$ Maria Elisabete Amaral de Moraes ${ }^{a}$ \\ Manoel Odorico de Moraes ${ }^{\text {a }}$ \\ a Universidade Federal do Ceara, Ceara, $\mathrm{e}^{\mathrm{b}}$ Universidade Federal do Rio de Janeiro, Rio de Janeiro, Brazil
}

\section{Key Words}

Thalidomide hybrid • Inflammatory corneal angiogenesis • Rabbits

\begin{abstract}
Aims: Evaluate the effect of LASSBio-596, structurally designed as a new hybrid of thalidomide, on inflammatory corneal angiogenesis. Methods: Eighteen rabbits were submitted to an alkaline cauterization in the right cornea. The animals were randomly allocated to three groups: vehicle, dexamethasone and LASSBio-596. Drugs were administered by eyedrops 3 times a day for 21 days. Evaluations were performed on days 3, 6, 9, 12, 15, 18 and 21 after cauterization. At these time points, digital images of the cornea were captured in a standard fashion. The angiogenic response was measured using software that was developed specifically for this purpose. It calculated the following parameters: neovascularization area (NA), total vascular length (TVL) and blood vessel number (BVN). Results: It was observed that dexamethasone significantly decreased NA, TVL and BVN during all assessments. From the NA the angiogenesis rate (AR) was calculated in each group. Therefore, dexamethasone completely inhibited the inflamma-
\end{abstract}

tory corneal angiogenesis with an AR of $-0.001 \pm 0.006$ $\mathrm{mm}^{2} /$ day, which was significantly lower $(\mathrm{p}<0.001)$ than that observed after treatment with vehicle $\left(0.078 \pm 0.024 \mathrm{~mm}^{2} /\right.$ day) and LASSBio-596 (0.054 $\pm 0.012 \mathrm{~mm}^{2} /$ day). Although LASSBio-596 reduced angiogenesis in relation to vehicle, according to NA, TVL and BVN values, this difference was not statistically significant. However, it was found that the AR as measured in the LASSBio-596 group was significantly lower $(p<0.05)$ than that seen in control animals, indicating a potential antiangiogenic effect. Conclusion: We conclude that topical application of LASSBio-596 at $1.0 \%$ has a potential inhibitory effect on inflammatory corneal angiogenesis in rabbits.

Copyright $\odot 2012$ S. Karger AG, Basel

\section{Introduction}

Angiogenesis or neovascularization is the development of new capillaries from preexisting blood vessels $[1,2]$. Several regulatory mechanisms of angiogenesis control both physiological processes and the spread of diseases [3]. Abnormal angiogenesis contributes to the pathogenesis of angiogenesis-dependent diseases such

\section{KARGER}

Fax +41613061234

E-Mail karger@karger.ch

www.karger.com
(C) 2012 S. Karger AG, Basel

0030-3747/12/0484-0177\$38.00/0

Accessible online at:

www.karger.com/ore
Joao Crispim M.L. Ribeiro

Al. Jurupis, 800/71A

Sao Paulo, SP 04088-002 (Brazil)

Tel. +551182376677

E-Mail jcmlribeiro@gmail.com 
as inflammatory disorders [4]. During the inflammatory process, the release of chemokines recruits leukocytes that produce angiogenic factors, such as vascular endothelial growth factor (VEGF), fibroblast growth factor 2 (FGF-2) and tumor necrosis factor $\alpha$ (TNF- $\alpha$ ), among others. These growth factors attract endothelial cells, smooth muscle cells, fibroblasts, leukocytes and platelets, triggering the process of neovascularization [5].

Corneal neovascularization from the limbic vascular plexus is a common feature of several diseases of the cornea, either of infectious, traumatic, degenerative or inflammatory origin [6-8]. The main stimuli for neovascularization are inflammation, hypoxia and limbic stem cell destruction [9]. There is exacerbation of the angiogenic process in various other diseases, namely diabetic retinopathy, macular degeneration and solid tumors [49]. For the treatment of these diseases, several drugs with antiangiogenic properties were identified, and their mechanisms of action were studied. Thalidomide is a potent inhibitor of angiogenesis caused by TNF- $\alpha$ and FGF2. However, due to its teratogenic effect in humans, its use is restricted in medical practice, engendering interest in the discovery of similar potent antiangiogenic analogs without this adverse effect [4].

LASSBio-596, structurally designed as a new hybrid of thalidomide and arylsulfonamide derivatives, is an agent that displays important anti-inflammatory and immunomodulatory properties [10-12]. The compound is obtained through the hydrolysis of the phthalimide ring, which is present in the structure of prototype LASSBio-468. But LASSBio-596 is considered safer than thalidomide because of the absence of phthalimide and glutarimide subunits, which are present in thalidomide and possibly responsible for its teratogenic profile (fig. 1). Modulation of the inflammatory process, as previously described, inhibits the recruitment of leukocytes induced by lipopolysaccharide, an effect that is accompanied by reduced levels of TNF- $\alpha$ [11]. The aim of this work is to evaluate the effect of the drug LASSBio-596 in a model of inflammatory corneal angiogenesis.

\section{Materials and Methods}

\section{Animals}

The study was conducted in accordance with federal and institutional guidelines approved by the Ethical Committee in Animal Research at the Federal University of Ceara (UFC) and with adherence to the tenets of the NIH Statement for the Use of Animals in Research. Male, white New Zealand rabbits aged between

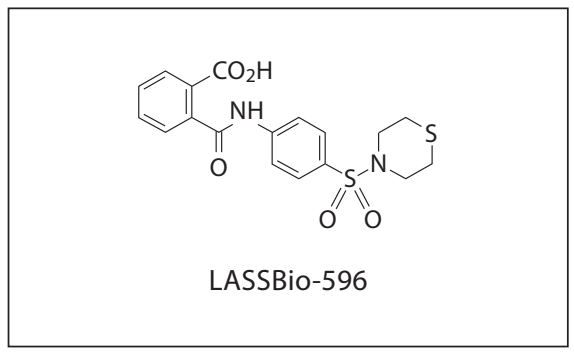

Fig. 1. Chemical structure of LASSBio-596.

90 and 120 days and weighing from 1,800 to 2,700 g were used. Rabbits were from the Department of Animal Science of the UFC and had previously been examined to exclude the possibility of external eye diseases. Rabbits were housed in cages designed specifically for their species. They remained in an air-conditioned environment, with appropriate conditions of temperature, humidity and lighting, with cycles of light and dark, alternating every $12 \mathrm{~h}$. Water and food were offered ad libitum. The latter consisted of a balanced diet for rabbits (Fri-Rabbit ${ }^{\circledR}$ ). The animals were subjected to an adjustment period of at least 7 days prior to the procedures.

\section{Experimental Eyedrops}

LASSBio-596 was synthesized by the Laboratory of Evaluation and Synthesis of Bioactive Substances (LASSBio ${ }^{\circledR}$ ) at the Federal University of Rio de Janeiro. In this study, LASSBio-596 had to be administered in an aqueous solution for efficacy as an eyedrop. The drug solubilization process was performed in the Department of Chemistry at the UFC. At first, a $1.0 \mathrm{wt} \%(\mathrm{w} / \mathrm{v})$ solution of LASSBio-596, made with benzalkonium chloride $(0.1 \% \mathrm{w} / \mathrm{v})$, was subjected to slow magnetic stirring for $5 \mathrm{~min}$. To this solution was added the block copolymer of ethylene oxide and $\varepsilon$ caprolactone, E114CL20 (where E represents an oxyethylene unit and CL $\varepsilon$-caprolactone; $1.0 \% \mathrm{w} / \mathrm{v})$ and Tween $80(0.02 \% \mathrm{w} / \mathrm{v})$. The homogenization was conducted on an Ultrasonic-Homogenizer Sonifier II W-450 (70 W, 0 cycle, Ms73 tip, 60 s) with the sample cooled in an ice bath. The sample was finally filtered through a $0.45-\mu \mathrm{m}$ membrane filter (Millex-HA Filter Units, Millipore ${ }^{\mathrm{TM}}$ ). To control for any confounding effect of the LASSBio-596 vehicle on ocular shedding, a vehicle eyedrop (a solution of benzalkonium chloride $0.1 \%$ added to the block copolymer E114CL20 1.0\% and Tween $800.02 \%$ ) served as control. Dexamethasone eyedrop $40 \mathrm{mg} / \mathrm{ml}$ (4.0\%) was prepared in distilled water and added to the solution of benzalkonium chloride $0.1 \%$.

\section{Inflammatory Corneal Angiogenesis Assay}

We used the model of inflammatory corneal angiogenesis induced by punctual alkaline cauterization, by which neovascularization is induced by an inflammatory response, i.e. a neovascular nonspecific stimulus [13]. Prior to cauterization and all subsequent assessments, the animals were anesthetized with a combination of $12 \mathrm{mg} / \mathrm{kg}$ ketamine hydrochloride and $4 \mathrm{mg} / \mathrm{kg}$ xylazine hydrochloride i.m., complemented by 2 drops of $0.5 \%$ proxymetacaine hydrochloride applied topically to the conjunctival sac. The anesthetized animal was placed in a Styrofoam tray in left lateral 
decubitus with the dorsum toward the examiner. Subsequently the eyelids were held apart with a blepharostat, exposing almost the entire corneal surface and the upper limbal area. The rabbits were submitted to a punctual cauterization on the top periphery of the right cornea with the aid of a microscope, using a magnification of $\times 16$. For this, we used a circular piece of filter paper ( $3 \mathrm{~mm}$ diameter), previously soaked in a solution of sodium hydroxide $(\mathrm{NaOH}) 1 \mathrm{M}$ for $30 \mathrm{~s}$ (excess solution was blotted away with gauze). The filter paper was left for $2 \mathrm{~min}$ at approximately $1 \mathrm{~mm}$ from the conjunctival limbal tissue in the 12-o'clock position near the insertion of the upper rectus muscle. The eye was subsequently rinsed with $5 \mathrm{ml}$ sodium chloride at $0.9 \%$ solution to remove excess $\mathrm{NaOH}$. The technique produced a $3.5-\mathrm{mm}$-wide, homogeneous and circular cauterization site with well-defined borders. The animals were returned to their cages only after complete emergence from anesthesia.

\section{Experimental Design}

The experiments were performed at the Laboratory of Experimental Surgery at the UFC. The rabbits were randomly allocated to three treatment groups: a vehicle group, consisting of 6 rabbits that were treated with 1 drop ( $40 \mu \mathrm{l}$ ) of the vehicle eyedrop, instilled in the conjunctival sac 3 times per day; a dexamethasone group, consisting of 6 rabbits that were treated with topical instillation of 1 drop $(40 \mu \mathrm{l})$ of dexamethasone $4.0 \%$ eyedrop 3 times per day; a LASSBio-596 group, composed of 6 rabbits that were treated with 1 drop $(40 \mu \mathrm{l})$ of the drug LASSBio-596 (diluted in solution at a concentration of $1.0 \%$ ) in the conjunctival sac 3 times per day. Treatments were initiated on the day of cauterization (day zero) and continued for 21 days.

\section{Image Acquisition and Processing}

The animals were evaluated on days 3, 6, 9, 12, 15, 18 and 21 following cauterization. During exposure the cornea was kept hydrated with frequent instillations of liquid ocular gel based on polyacrylic acid, a lacrimal fluid substitute. Images of the cornea including the area of angiogenesis were then acquired in standard fashion with a video camera coupled to a surgical microscope that transferred data to a microcomputer. The microscope axis was positioned perpendicularly to the tangent of the corneal periphery to minimize spatial distortion. In addition, a green filter was used to increase the contrast of the blood vessels. Images were acquired at $\times 25$ magnification and saved as Windows ${ }^{\circledR}$ bitmaps measuring $320 \times 240$ pixels.

The angiogenesis response was measured by the Angiogenesis Quantifier System, a software developed specifically for this purpose [13]. The system processed the digital images of the cornea containing the area of angiogenesis and determined the following parameters: neovascularization area (NA), total vessel length (TVL) and blood vessel number (BVN) (fig. 2). From the NA, it was possible to calculate the angiogenesis rate (AR) and the inhibitory effect (IE) of each treatment in relation to vehicle at day 21 using the following equations:

$$
\begin{aligned}
& A R=\frac{N A(\text { day } 21)-N A(\text { day } 3)}{18} \\
& I E(\text { drug })=\frac{N A(\text { vehicle })-N A(\text { drug })}{N A(\text { vehicle })} \times 100
\end{aligned}
$$

Evaluation of these parameters provided spatial and temporal tracking of the angiogenic response in vivo. Then a quantitative analysis of the inhibitory effect of each substance was performed.

\section{Statistical Analysis}

Quantitative variables were initially examined by the Kolmogorov-Smirnov test to verify normality of distribution. Mean and standard deviation were then calculated as descriptive statistics. Parametric tests were used for data analysis. To compare the three treatment groups, we used analysis of variance associated with Tukey's multiple comparison test. The unpaired t test was used to compare two variables that were not matched. For matched variables, comparisons between paired variables were performed using the $t$ test. In all cases, the level of statistical significance was set at $<0.05$. For data analysis, as well as for preparation of graphics, we used the statistical software Graph Pad Prism ${ }^{\circledR}$ version 5.00 for Windows ${ }^{\circledR}$ (Graph Pad Software, San Diego, Calif., USA, 2007).

\section{Results}

The evolution of the angiogenesis parameters (NA, TVL and BVN) in the vehicle group reveals a biphasic pattern characterized by a period of intense vascular growth up to day 12 , followed by a period of stability up to day 21 (fig. 3,4 ). The maximum NA, TVL and BVN values recorded were $1.998 \pm 0.622 \mathrm{~mm}^{2}$ (day 21), 31.956 $\pm 10.535 \mathrm{~mm}$ (day 21) and $205.333 \pm 54.503$ vessels (day 18).

In the dexamethasone group, there was a significant reduction $(\mathrm{p}<0.05)$ compared with vehicle on day 3 in NA, TVL and BVN. During all subsequent assessments, the parameters for dexamethasone decreased further $(\mathrm{p}<0.001)$ in comparison with the vehicle group (fig. 3 , 4). The maximum NA, TVL and BVN values were 0.229 $\pm 0.089 \mathrm{~mm}^{2}$ (day 18), $3.618 \pm 1.367 \mathrm{~mm}$ (day 18) and $26.000 \pm 20.396$ vessels (day 9).

With regard to the antiangiogenic effect of the drug in this study, LASSBio-596 elicited the same trend as vehicle during the first 6 days, which was true for all the parameters evaluated. From the sixth day, LASSBio-596 reduced angiogenesis progression as compared with vehicle. This trend was maintained until the end of the experiment. However, the observed reduction was not statistically significant. All parameters remained constant in the LASSBio-596 group from the sixth day onward (fig. 3,4$)$. The maximum NA, TVL and BVN values recorded were $1.680 \pm 0.322 \mathrm{~mm}^{2}$ (day 18), $27.202 \pm$ $6.216 \mathrm{~mm}$ (day 15) and $178.667 \pm 44.889$ vessels (day 15), respectively.

The vascular growth rate was measured throughout the study as AR (fig. 5). In the dexamethasone group, we 


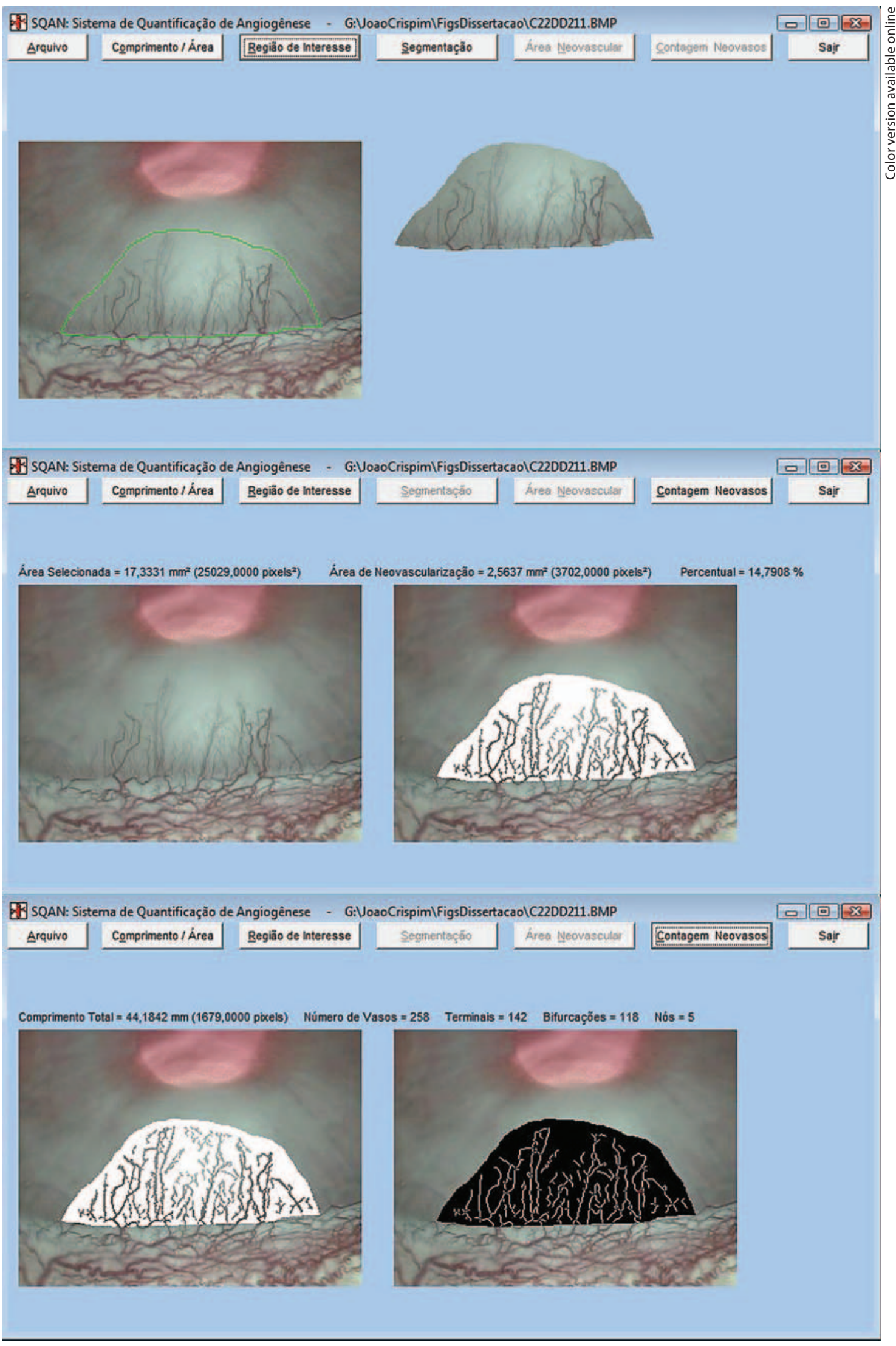



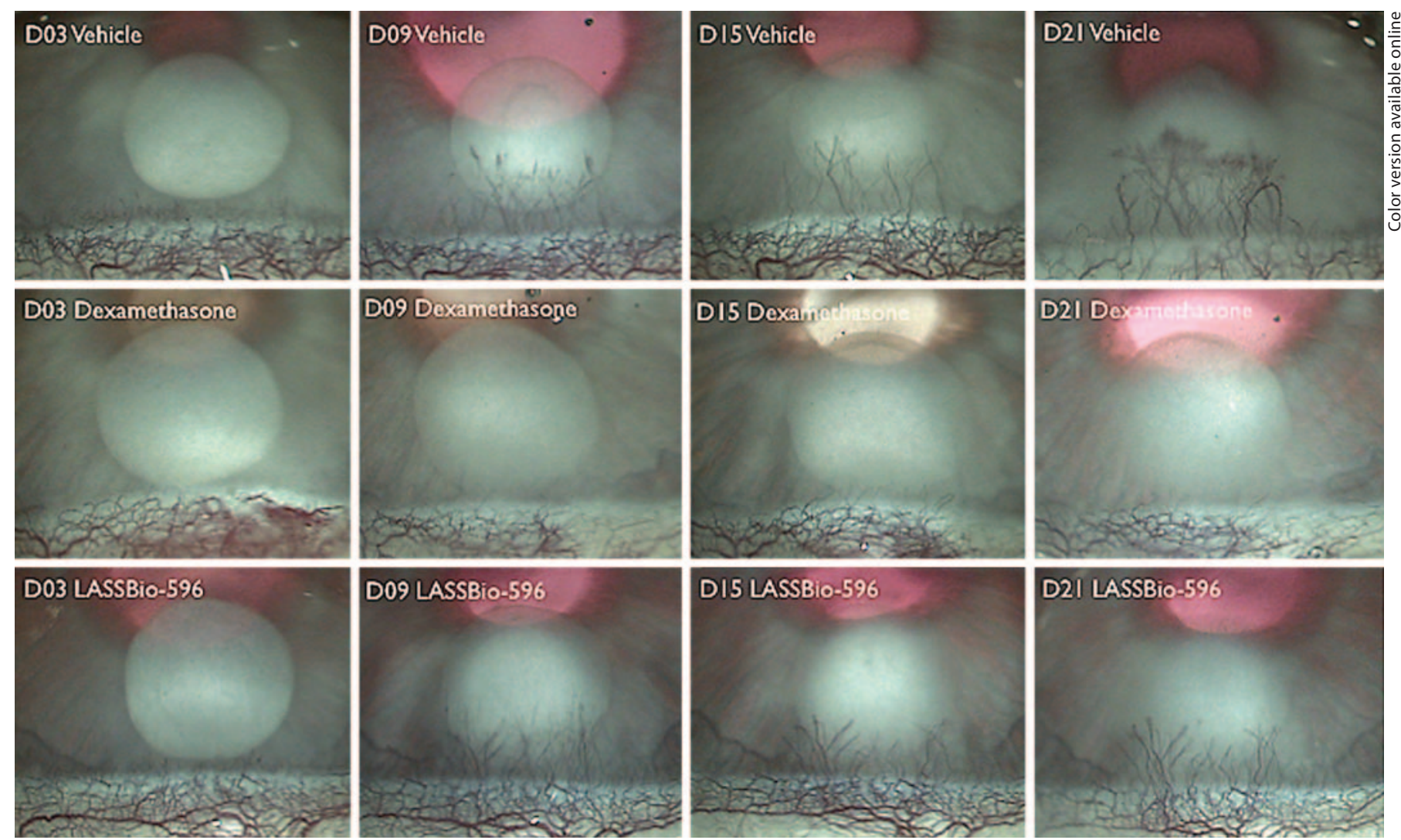

Fig. 3. Angiogenic response observed in experimental groups: vehicle, dexamethasone and LASSBio-596. Images acquired from days 3, 9, 15 and 21 after cauterization. Magnification $\times 25$.

observed a complete inhibition of the inflammatory corneal angiogenesis with a negative AR $(-0.001 \pm 0.006$ $\mathrm{mm}^{2} /$ day), which was significantly lower $(\mathrm{p}<0.001)$ than vehicle $\left(0.078 \pm 0.024 \mathrm{~mm}^{2} /\right.$ day $)$ and LASSBio-596 $(0.054$ $\pm 0.012 \mathrm{~mm}^{2} /$ day $)$.

Moreover, the AR measured in the LASSBio-596 group $\left(0.054 \pm 0.012 \mathrm{~mm}^{2} /\right.$ day) was significantly lower $(\mathrm{p}<$ $0.05)$ than that seen in the vehicle group $(0.078 \pm 0.024$ $\mathrm{mm}^{2} /$ day), suggesting a potential antiangiogenic effect, mainly during the second half of the angiogenic process (fig. 5).

It is noteworthy that no adverse effects were observed in the animal groups evaluated.

Fig. 2. Measurement of the angiogenesis response by the Angiogenesis Quantifier System (SQAN). First of all, it determined the region of interest, then it calculated the paramenters: neovascularization area (NA), total vessel length (TVL) and blood vessel number (BVN).

Effect of LASSBio-596 on Inflammatory Corneal Angiogenesis

\section{Discussion}

Our main purpose was not to investigate the cellular and molecular events involved in corneal neovascularization but to study the kinetics of the angiogenic process. Thus, according to the angiogenesis measured, we can infer that the temporal progression of the angiogenesis response followed a biphasic pattern, correlating with the phases of proliferation and maturation observed in a model of inflammatory neovascularization in the murine cornea [14]. The first phase is characterized by intense vascular proliferation. Vascular density is high, there are numerous vascular buds, and the rate of angiogenesis reaches maximum values. The second phase is characterized by vascular maturation. The new vessels lengthen with reduced branching and the rate of angiogenesis is stabilized. Therefore, this is a better representation of the phenomenon under study.

Several studies have demonstrated the antiangiogenic activity of glucocorticoids in models of corneal angiogen-

Ophthalmic Res 2012;48:177-185 


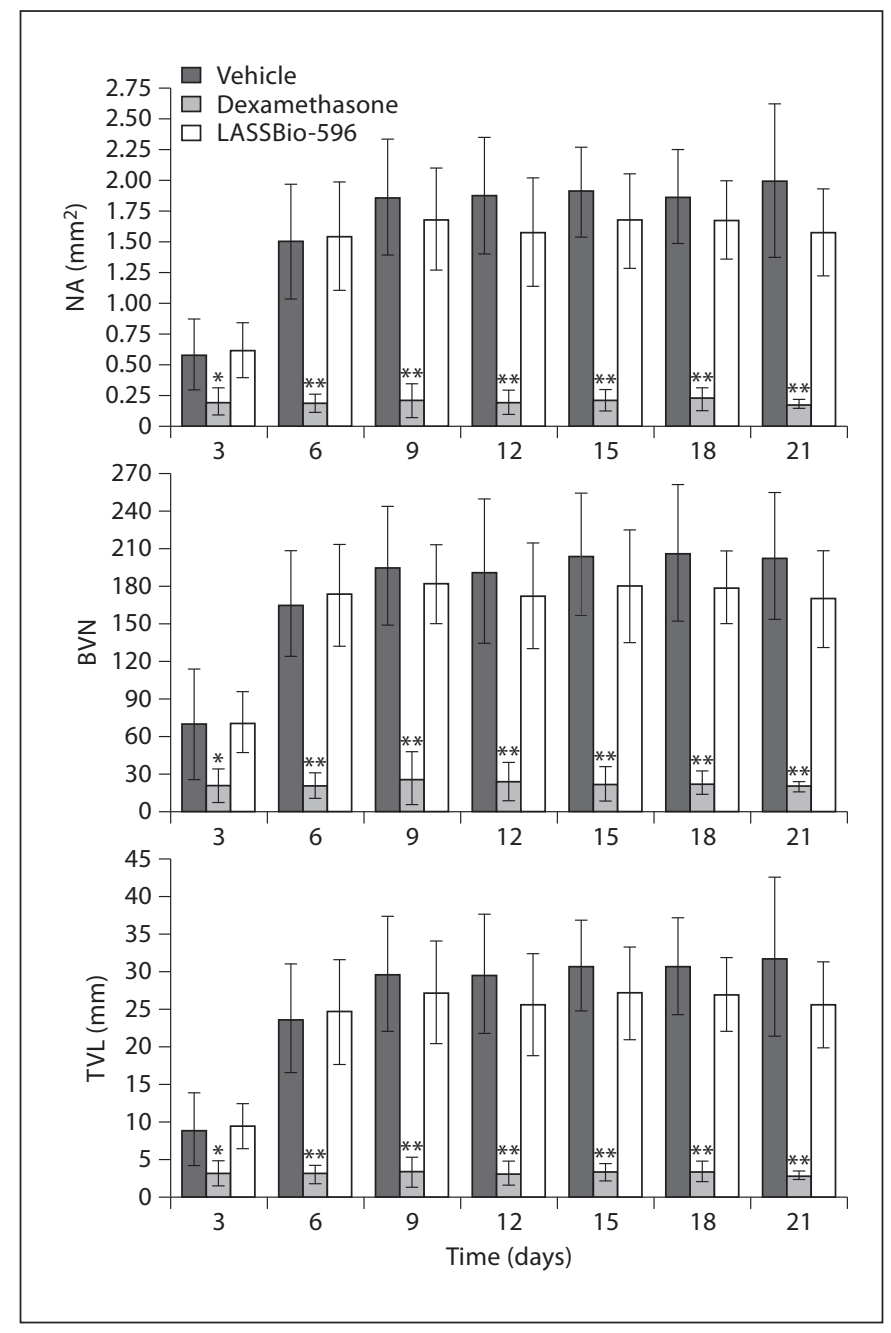

Fig. 4. Quantification of angiogenic response in the vehicle, dexamethasone and LASSBio-596 groups, based on NA, BVN and TVL parameters. The data, expressed in millimeters, correspond to the mean and standard deviation of measurements made on 6 animals in each group on days $3,6,9,12,15,18$ and 21 after cauterization. To compare the three treatment groups on the same day, we used the analysis of variance associated with Tukey's multiple comparison test, which showed differences between paired groups. ${ }^{*} \mathrm{p}<0.05,{ }^{* *} \mathrm{p}<0.01$.

esis induced by both inflammation and by specific factors. In fact, glucocorticoids and nonsteroidal anti-inflammatory drugs constitute the main pharmacological approaches to the treatment of corneal neovascularization [15]. Dexamethasone is widely used in the treatment of corneal inflammation. Several authors had previously showed the antiangiogenic effect of dexamethasone, including on cauterization-induced corneal neovascularization [16-19]. In 1987, Folkman and Ingber [16] report-

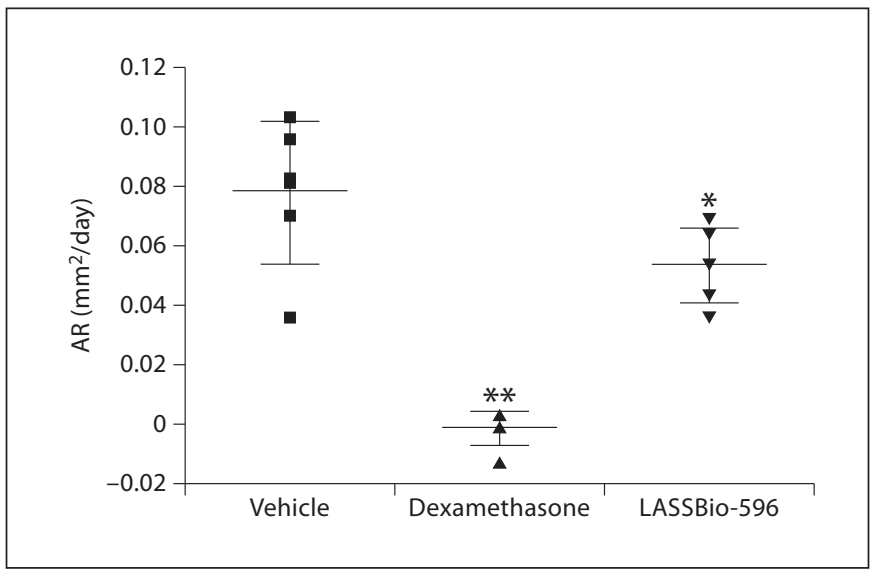

Fig. 5. AR in square millimeters per day, for the period between days 3 and 21, calculated for the vehicle, dexamethasone and LASSBio-596 groups. The data represent the mean and standard deviation of measurements made on 6 animals in each group. The analysis of variance was used to compare the three treatment groups, supplemented by Tukey's multiple comparison test, which showed differences between paired groups. ${ }^{*} \mathrm{p}<0.05,{ }^{* *} \mathrm{p}<0.01$.

ed the antiangiogenic function of a class of steroids, including dexamethasone, which they named the angiostatic steroids. However, because of its side effects, including cataract and intraocular hypertension, steroid therapy in the management of some corneal diseases remains controversial. Dexamethasone blocks the transcription of inflammatory proteins by prohibiting the activity of the nuclear factor $\kappa B(N F-\kappa B)$ [18]. NF- $\kappa B$ plays an important role in interleukin (IL)-1 $\beta$-related inflammatory diseases, including various corneal diseases. Nakao et al. $[19]$ showed that IL-1 $\beta$-induced NF- $\kappa B$ activation is inhibited by dexamethasone and that a selective NF- $\kappa$ B inhibitor diminished inflammatory corneal angiogenesis. In our study, dexamethasone eyedrops $(4.0 \%)$ were administered topically 3 times a day and fully inhibited the angiogenic response throughout the experiment. In the dexamethasone group, NA, TVL and BVN were significantly $(p<0.05)$ reduced compared with vehicle on day 3 . In all subsequent evaluations, there was a further decrease $(\mathrm{p}<0.001)$ compared with vehicle (fig. 3, 4). Thus, dexamethasone completely inhibited the inflammatory corneal angiogenesis, yielding a negative AR $(-0.001 \pm$ $0.006 \mathrm{~mm}^{2} /$ day $)$ that was significantly lower $(\mathrm{p}<0.001)$ than that observed with vehicle $\left(0.078 \pm 0.024 \mathrm{~mm}^{2} /\right.$ day $)$ and LASSBio-596 (0.054 $\pm 0.012 \mathrm{~mm}^{2} /$ day; fig. 5). It should be emphasized that, although the inflammatory response has not been assessed from the histological 
point of view, visual inspection of animals treated with dexamethasone revealed that signs of inflammation were much less intense in treated animals compared with those that received vehicle.

Thalidomide, a synthetic derivative of glutamic acid, is a drug with immunomodulatory, anti-inflammatory, antiangiogenic and antitumoral properties [20-24]. In addition to its inhibitory effect on corneal neovascularization induced by VEGF and FGF-2, the bioactivity of thalidomide is related to its ability to inhibit the production of TNF- $\alpha$, a proangiogenesis and proinflammatory cytokine produced by monocytes/macrophages [18-20]. It is likely that the activity of thalidomide is in part controlled by NF- $\kappa$ B. Notably, TNF- $\alpha$ is one of the inducers of NF- $\kappa \mathrm{B}$ activation. NF- $\kappa \mathrm{B}$ regulates the expression of several genes involved in cell proliferation, inflammation, angiogenesis and the inhibition of apoptosis. Two of the genes regulated by activation of NF- $\kappa \mathrm{B}$ induced by TNF- $\alpha$ express the proangiogenic factors VEGF and IL8. Thus, it was postulated that thalidomide would block the activation of NF- $\kappa$ B induced by TNF- $\alpha$. Its antiangiogenic effect would result from inhibition of VEGF, IL-8 and other angiogenic factors regulated by NF- $\mathrm{B}$. Other proangiogenic factors are also inhibited by thalidomide - for example IL-6, a potent growth factor for malignant cells [20].

Initially introduced as a sedative, thalidomide was subsequently withdrawn from the market due to its teratogenicity, causing malformation of limbs in newborns, a condition denoted as phocomelia. Years later, D'Amato et al. [24] postulated that this malformation resulted from the inhibition of vascular growth in the developing buds of the members, during the embryonic period. With the discovery of its potent anti-inflammatory and immunomodulatory effects, thalidomide is currently used in certain pathologies with rigorous restrictions and control. So the search for new thalidomide analogs that combine high-power antiangiogenic and anti-inflammatory effects without severer adverse effects is an important goal.

Recently, LASSBio-596 was reported as a new anti-inflammatory and antifibrotic drug candidate, originally designed to mimic thalidomide and arylsulfonamide hybrids. The absence in the structure of LASSBio-596 of the two subunits that compose the thalidomide structure (i.e. phthalimide and glutarimide rings) yields a drug that is free of teratogenic effects [10].

Previously, in a model of pulmonary inflammation, LASSBio-596 demonstrated potent inhibitory activity in the recruitment of leukocytes induced by lipopolysaccharide, correlating with its effect in attenuating the levels of
TNF- $\alpha$ [11]. Demonstrating potent inhibitory activity in the recruitment of leukocytes induced by lipopolysaccharide, LASSBio-596 also attenuated the levels of TNF- $\alpha$ during pulmonary inflammation, which aroused our interest in studying its role in inflammatory angiogenesis. We hypothesized that the drug exerted an antiangiogenic effect. Moreover, LASSBio-596 was reported to effectively prevent mechanical and morphometric changes in the lung, and it was observed to block fibroproliferation in a BALB/c mouse model of asthma [12]. It was also reported that LASSBio-596 prevented lung and hepatic inflammation and completely blocked pulmonary functional and morphological changes induced by microcystins $[25,26]$. Because of this important anti-inflammatory action, we begin the study of LASSBio-596 in inflammatory angiogenesis, believing in its potential antiangiogenic effect.

In this study, the values of NA, TVL and BVN quantifiers in the LASSBio-596 group were similar to those in the vehicle group during the first 6 days of the experiment. From the sixth day, LASSBio-596 reduced proliferation of new vessels compared with vehicle; this effect was maintained until the end of the experiment, although the difference was not significant. It was also observed that all parameters remained constant in the LASSBio-596 group from the sixth day (fig. 3, 4). By visual inspection, the decrease on vascular thickness was noteworthy in the LASSBio-596 group compared with the vehicle group (fig. 3). However, analyzing the AR in the LASSBio-596 group $\left(0.054 \pm 0.012 \mathrm{~mm}^{2} /\right.$ day $)$, we observed a significant reduction $(\mathrm{p}<0.05)$ compared with the vehicle group $\left(0.078 \pm 0.024 \mathrm{~mm}^{2} /\right.$ day $)$. These findings demonstrate the drug's potential inhibitory effect on angiogenesis, mainly during the second half of the angiogenic process (fig. 5 ).

In evaluating the effectiveness of the final treatments, it was observed that dexamethasone had an inhibitory effect of $90.81 \%$, calculated on the basis of data from the vehicle group on day 21. These findings confirmed the drug's potent antiangiogenic effect. Regarding LASSBio-596, there was an inhibitory effect of $20.74 \%$ compared with the vehicle group. Despite the lower inhibitory effect of LASSBio-596 compared with dexamethasone, we can expect an inhibitory effect because it is a thalidomide hybrid, which probably exhibits analog properties. D'Amato et al. [24] investigated the inhibitory effect of thalidomide and its analogs in rabbits using a model based on angiogenesis induced by pellets containing FGF-2. Their results showed a significant inhibitory effect of thalidomide, despite the fact that the nonterato- 
genic analog supidimide has not presented any antiangiogenic activity. Furthermore, Joussen et al. [27] correlated the antiangiogenic effect of thalidomide and its derivatives supidimide and EM12 in a model of corneal neovascularization induced by VEGF and basic FGF with its potent teratogenic effects. They concluded by stating that further emphasis should be given to the investigation of thalidomide analogs that topically have the potential antiangiogenic effect. According to our results, we observed the potential inhibitor effect of LASSBio-596 on neovascularization, verifying that its AR was significantly lower than that of the vehicle group $(\mathrm{p}<0.05)$.

Looking retrospectively into the possible limitations of the study, we report the difficulty during the initial dilution of the drug under study, LASSBio-596. It was difficult to obtain a collyrium, necessary to maximize the drug's effect by topical application in the cornea. Furthermore, we did not investigate treatment with other concentrations to establish dose-response curves. We believe that the next studies might reveal promising results, including unmasking LASSBio-596's molecular mechanism of action in angiogenesis.
According to the presented data, we concluded that LASSBio-596, a new hybrid of thalidomide, had a potential inhibitory effect, as determined by topical application at $1.0 \%$, on inflammatory corneal angiogenesis in rabbits. At this time, we present initial results demonstrating the potential inhibitory effect of LASSBio-596 on inflammatory corneal angiogenesis. We would like to express our interest in continuing to study the effect of this new thalidomide hybrid in other experimental models involving neovascularization, allowing a greater understanding of its effect on the process of angiogenesis. Additionally, we would like to contribute to the science related to the discovery of new drugs that are capable of assisting in the fight against angiogenesis-dependent diseases.

\section{Acknowledgements}

We would like to acknowledge everyone who contributed to the development of the research, especially the Postgraduate Program of the Department of Surgery from the UFC.

\section{References}

$>1$ Hanahan D, Folkman J: Patterns and emerging mechanisms of the angiogenic switch during tumorigenesis. Cell 1996;86:353364.

$>2$ Auerbach R, Lewis R, Shinners B, Kubai L, Akhtar N: Angiogenesis assays: a critical overview. Clin Chem 2003;49:32-40.

>3 Papetti M, Herman IM: Mechanisms of normal and tumor-derived angiogenesis. Am J Physiol Cell Physiol 2002;282:947-970.

4 Folkman J: Seminars in Medicine of the Beth Israel Hospital, Boston. Clinical applications of research on angiogenesis. N Engl J Med 1995;333:1757-1763.

$\checkmark 5$ Carmeliet P: Mechanisms of angiogenesis and arteriogenesis. Nat Med 2000;6:389395.

6 Joussen AM, Beecken WD, Moromizato Y, Schwartz A, Kirchhof B, Poulaki V: Inhibition of inflammatory corneal angiogenesis by TNP-470. Invest Ophthalmol Vis Sci 2001;42:2510-2516.

7 Yoshida S, Yoshida A, Matsui H, Takada Y, Ishibashi T: Involvement of macrophage chemotactic protein-1 and interleukin-1beta during inflammatory but not basic fibroblast growth factor-dependent neovascularization in the mouse cornea. Lab Invest 2003;83: 927-938.
>8 Poulaki V, Mitsiades N, Kruse FE, Radetzky S, Iliaki E, Kirchhof B, et al: Activin a in the regulation of corneal neovascularization and vascular endothelial growth factor expression. Am J Pathol 2004;164:1293-1302.

$\checkmark 9$ Adamis AP, Aiello LP, D’Amato RA: Angiogenesis and ophthalmic disease. Angiogenesis 1999;3:9-14.

10 Lima LM, Castro P, Machado AL, Fraga CA, Lugnier C, de Moraes VL, et al: Synthesis and anti-inflammatory activity of phthalimide derivatives, designed as new thalidomide analogues. Bioorg Med Chem 2002;10:30673073.

11 Rocco PR, Momesso DP, Figueira RC, Ferreira $\mathrm{HC}$, Cadete RA, Legora-Machado A, et al: Therapeutic potential of a new phosphodiesterase inhibitor in acute lung injury. Eur Respir J 2003;22:20-27.

12 Campos HS, Xisto DG, Oliveira MB, Teixeira I, Negri EM, Mauad T, et al: Protective effects of phosphodiesterase inhibitors on lung function and remodeling in a murine model of chronic asthma. Braz J Med Biol Res 2006;39:283-287.

13 Fechine-Jamacaru FV, Fechine Junior JU, de Moraes Filho MO: Model of inflammatory angiogenesis in rabbit cornea induced by punctual alkaline cauterization. Acta Cir Bras 2005;20:64-73.
14 Edelman JL, Castro MR, Wen Y: Correlation of VEGF expression by leukocytes with the growth and regression of blood vessels in the rat cornea. Invest Ophthalmol Vis Sci 1999; 40:1112-1123.

15 Usui T, Sugisaki K, Amano S, Jang WD, Nishiyama N, Kataoka K: New drug delivery for corneal neovascularization using polyion complex micelles. Cornea 2005;24:39-42.

16 Folkman J, Ingber DE: Angiostatic steroids. Method of discovery and mechanism of action. Ann Surg 1987;206:374-383.

17 Proia AD, Hirakata A, McInnes JS, Scroggs MW, Parikh I: The effect of angiostatic steroids and $\beta$-cyclodextrin tetradecasulfate on corneal neovascularization in the rat. Exp Eye Res 1993;57:693-698.

18 Rhen T, Cidlowski JA: Antiinflammatory action of glucocorticoids - new mechanisms for old drugs. N Engl J Med 2005;353:17111723.

19 Nakao S, Hata Y, Miura M, Noda K, Kimura YN, Kawahara S, et al: Dexamethasone inhibits interleukin-1 $\beta$-induced corneal neovascularization: role of nuclear factor- $\kappa \mathrm{B}$ activated stromal cells in inflammatory angiogenesis. Am J Pathol 2007;171:1058-1065. 
20 Macpherson GR, Franks M, Tomoaia-Cotisel A, Ando Y, Price DK, Figg WD: Current status of thalidomide and its role in the treatment of metastatic prostate cancer. Crit Rev Oncol Hematol 2003;46:49-57.

-21 Eleutherakis-Papaiakovou V, Bamias A, Dimopoulos MA: Thalidomide in cancer medicine. Ann Oncol 2004;15:1151-1160.

22 Kenyon BM, Browne F, D’Amato RJ: Effects of thalidomide and related metabolites in a mouse corneal model of neovascularization. Exp Eye Res 1997;64:971-978.
23 Gasparini G, Longo R, Fanelli M, Teicher BA: Combination of antiangiogenic therapy with other anticancer therapies: results, challenges, and open questions. J Clin Oncol 2005;23:1295-1311.

24 D’Amato RJ, Loughnan MS, Flynn E, Folkman J: Thalidomide is an inhibitor of angiogenesis. Proc Natl Acad Sci USA 1994;91: 4082-4085.

25 Carvalho GMC, Oliveira VR, Soares RM, Azevedo SMFO, Lima LM, Barreiro EJ, et al: Can LASSBio 596 and dexamethasone treat acute lung and liver inflammation induced by microcystin-LR? Toxicon 2010;56:604612 .
26 Casquilho NV, Carvalho GMC, Alves JLCR, Machado MN, Soares RM, Azevedo SMFO, et al: LASSBio 596 per os avoids pulmonary and hepatic inflammation induced by $\mathrm{mi}$ crocystin-LR. Toxicon 2011;58:195-201.

27 Joussen AM, Germann T, Kirchhof B: Effect of thalidomide and structurally related compounds on corneal angiogenesis is comparable to their teratological potency. Graefes Arch Clin Exp Ophthalmol 1999;237:952961. 